\title{
The general meromorphic solutions of the Petviashvili equation
}

\author{
Zifeng Huang ${ }^{1,2}$, Wenjun Yuan ${ }^{1 *}$ and Jinchun Lai ${ }^{1}$
}

\section{*Correspondence:}

wjyuan1957@126.com

'School of Mathematics and Information Science, Guangzhou

University, Guangzhou, 510006

China

Full list of author information is available at the end of the article

\begin{abstract}
In this paper, we employ the complex method to first obtain all meromorphic exact solutions of complex Petviashvili equation, and then find all exact solutions of Petviashvili equation. The idea introduced in this paper can be applied to other non-linear evolution equations. Our results show that the complex method is simpler than other methods. Finally, we give some computer simulations to illustrate our main results.
\end{abstract}

MSC: Primary 30D35; secondary 34A05

Keywords: Petviashvili equation; exact solution; meromorphic function; elliptic function

\section{Introduction and main results}

In 2006 and 2008, Zhang et al. [1,2] obtained abundant exact solutions of the Petviashvili equation by using the modified mapping method and the availability of symbolic computation. These solutions include the Jacobi elliptic function solutions, triangular function solutions, and soliton solutions. In this paper, we employ the complex method to obtain first all traveling meromorphic exact solutions of complex Petviashvili equation, and then find all exact solutions of the Petviashvili equation.

In order to state our main result, we need some concepts and some notation. A meromorphic function $w(z)$ means that $w(z)$ is holomorphic in the complex plane $\mathbb{C}$ except for poles. $\alpha, b, c, c_{i}$, and $c_{i j}$ are constants, which may be different from each other in different places. We say that a meromorphic function $f$ belongs to the class $W$ if $f$ is an elliptic function, or a rational function of $e^{\alpha z}(\alpha \in \mathbb{C})$, or a rational function of $z$.

The Petviashvili equation $[1,2]$ is

$$
\frac{\partial}{\partial t}\left(\nabla^{2} \phi-\phi\right)+C_{R}(1+\phi) \frac{\partial \phi}{\partial t}+J\left(\phi, \nabla^{2} \phi\right)=0,
$$

where

$$
\begin{aligned}
& \nabla^{2}=\frac{\partial}{\partial x^{2}}+\frac{\partial}{\partial y^{2}}, \quad J(A, B)=\frac{\partial A}{\partial x} \frac{\partial B}{\partial y}-\frac{\partial A}{\partial y} \frac{\partial B}{\partial x}, \\
& C_{R}=\frac{\beta L_{R}^{2}}{\sqrt{g H}}
\end{aligned}
$$

are two-dimensional Laplace and Jacobian operators, respectively, $C_{R}$ is the linear zerodimensional phase velocity of Rossby wave, $L_{R}$ is the characteristic length about $x$ and $y$,

(C2014 Huang et al.; licensee Springer. This is an Open Access article distributed under the terms of the Creative Commons Attribution License (http://creativecommons.org/licenses/by/2.0), which permits unrestricted use, distribution, and reproduction in any medium, provided the original work is properly cited. 
$H$ is the average thickness of the fluid, and $g$ is the acceleration of gravity; $\beta=\frac{\partial f}{\partial y}$, and $\frac{1}{f}$ is the characteristic value about $t$.

Substituting the traveling wave transformation

$$
\phi=w(z), \quad z=k x+l y-\omega t
$$

into the Petviashvili equation gives a non-linear ordinary differential equation

$$
-\omega\left(k^{2}+l^{2}\right) w^{\prime \prime \prime}+k C_{R} w w^{\prime}+\left(\omega+k C_{R}\right) w^{\prime}=0,
$$

and integrating it yields the auxiliary ordinary differential equation

$$
-\omega\left(k^{2}+l^{2}\right) w^{\prime \prime}+\frac{1}{2} k C_{R} w^{2}+\left(\omega+k C_{R}\right) w+d=0,
$$

where $\omega, k, l, C_{R}$ are constants.

Our main result is Theorem 1 .

Theorem 1 Equation (1) is integrable if and only if $2 d k C_{R}=-12 \omega^{2}\left(k^{2}+l^{2}\right)^{2} g_{2}+\left(\omega+k C_{k}\right)^{2}$, $F^{2}=4 E^{3}-g_{2} E-g_{3}, g_{3}$, and E are arbitrary. Furthermore, the general solutions of the Eq. (1) are of the following form.

(I) The elliptic general solutions are

$$
\begin{aligned}
w_{d}(z)= & \frac{12 \omega\left(k^{2}+l^{2}\right)}{k C_{R}}\left\{-\wp(z)+\frac{1}{4}\left[\frac{\wp^{\prime}(z)+F}{\wp(z)-E}\right]^{2}\right\} \\
& -\frac{12 \omega\left(k^{2}+l^{2}\right) E}{k C_{R}}-1-\frac{\omega}{k C_{R}},
\end{aligned}
$$

if $2 d k C_{R}=-12 \omega^{2}\left(k^{2}+l^{2}\right)^{2} g_{2}+\left(\omega+k C_{k}\right)^{2}, F^{2}=4 E^{3}-g_{2} E-g_{3}, g_{3}$ and $E$ are arbitrary.

(II) The simply periodic solutions are

$$
\begin{gathered}
w_{s}(z)=\frac{3 \omega\left(k^{2}+l^{2}\right)}{k C_{R}} \alpha \operatorname{coth}^{2} \frac{\alpha}{2}\left(z-z_{0}\right)-\frac{2 \omega\left(k^{2}+l^{2}\right)}{k C_{R}} \alpha^{2}-1-\frac{\omega}{k C_{R}}, \\
\text { if } 2 d k C_{R}=-\omega^{2}\left(k^{2}+l^{2}\right)^{2} \alpha^{4}+\left(\omega+k C_{k}\right)^{2}, \alpha \neq 0, z_{0} \in \mathbb{C} .
\end{gathered}
$$

(III) The rational function solutions are

$$
\begin{gathered}
w_{R}(z)=\frac{12 \omega\left(k^{2}+l^{2}\right)}{k C_{R}\left(z-z_{0}\right)^{2}}-1-\frac{\omega}{k C_{R}}, \\
\text { if } 2 d k C_{R}=\left(\omega+k C_{k}\right)^{2}, z_{0} \in \mathbb{C} .
\end{gathered}
$$

\section{Preliminary lemmas and the complex method}

In order to explain our complex method and give the proof of Theorem 1, we need some lemmas and results. 
Lemma $1[3,4]$ Let $k \in \mathbb{N}$, then any meromorphic solution $w$ with at least one pole of the $k$ th order Briot-Bouquet equation,

$$
F\left(w^{(k)}, w\right)=\sum_{i=0}^{n} P_{i}(w)\left(w^{(k)}\right)^{i}=0
$$

belongs to $W$, where $P_{i}(w)$ are polynomials in $w$ with constant coefficients.

Set $m \in \mathbf{N}:=\{1,2,3, \ldots\}, r_{j} \in \mathbf{N}_{0}=\mathbf{N} \cup\{0\}, r=\left(r_{0}, r_{1}, \ldots, r_{m}\right), j=0,1, \ldots, m$. Define

$$
M_{r}[w](z):=[w(z)]^{r_{0}}\left[w^{\prime}(z)\right]^{r_{1}}\left[w^{\prime \prime}(z)\right]^{r_{2}} \cdots\left[w^{(m)}(z)\right]^{r_{m}} .
$$

$p(r):=r_{0}+r_{1}+\cdots+r_{m}$ is called the degree of $M_{r}[w]$. The differential polynomial $P\left(w, w^{\prime}, \ldots, w^{(m)}\right)$ is defined as follows:

$$
P\left(w, w^{\prime}, \ldots, w^{(m)}\right):=\sum_{r \in I} a_{r} M_{r}[w]
$$

where $a_{r}$ are constants, and $I$ is a finite index set. The total degree of $P\left(w, w^{\prime}, \ldots, w^{(m)}\right)$ is defined by $\operatorname{deg} P\left(w, w^{\prime}, \ldots, w^{(m)}\right):=\max _{r \in I}\{p(r)\}$.

We will consider the following complex ordinary differential equations:

$$
P\left(w, w^{\prime}, \ldots, w^{(m)}\right)=b w^{n}+c,
$$

where $b \neq 0, c$ are constants, $n \in \mathbf{N}$.

Definition 2 Let $p, q \in \mathbf{N}$. Suppose that Eq. (5) has a meromorphic solution $w$ with at least one pole; then we say that Eq. (5) satisfies the weak $\langle p, q\rangle$ condition if substituting the Laurent series

$$
w(z)=\sum_{k=-q}^{\infty} c_{k} z^{k}, \quad q>0, c_{-q} \neq 0
$$

into Eq. (5) we can determine $p$ distinct Laurent singular parts in the form below:

$$
\sum_{k=-q}^{-1} c_{k} z^{k}
$$

Lemma 3 [5-7] Let $p, l, m, n \in \mathbf{N}, \operatorname{deg} P\left(w, w^{(m)}\right)<n$. Suppose that the mth order BriotBouquet equation

$$
P\left(w^{(m)}, w\right)=b w^{n}+c
$$

satisfies the weak $\langle p, q\rangle$ condition; then all meromorphic solutions $w$ belong to the class $W$. If for some values of parameters such a solution $w$ exists, then other meromorphic solutions 
form a one-parametric family $w\left(z-z_{0}\right), z_{0} \in \mathbf{C}$. Furthermore each elliptic solution with a pole at $z=0$ can be written as

$$
\begin{aligned}
w(z)= & \sum_{i=1}^{l-1} \sum_{j=2}^{q} \frac{(-1)^{j} c_{-i j}}{(j-1) !} \frac{d^{j-2}}{d z^{j-2}}\left(\frac{1}{4}\left[\frac{\wp^{\prime}(z)+B_{i}}{\wp(z)-A_{i}}\right]^{2}-\wp(z)\right) \\
& +\sum_{i=1}^{l-1} \frac{c_{-i 1}}{2} \frac{\wp^{\prime}(z)+B_{i}}{\wp(z)-A_{i}}+\sum_{j=2}^{q} \frac{(-1)^{j} c_{-l j}}{(j-1) !} \frac{d^{j-2}}{d z^{j-2}} \wp(z)+c_{0},
\end{aligned}
$$

where $c_{-i j}$ are given by Eq. (6), $B_{i}^{2}=4 A_{i}^{3}-g_{2} A_{i}-g_{3}$, and $\sum_{i=1}^{l} c_{-i 1}=0$.

Each rational function solution $w:=R(z)$ is of the form

$$
R(z)=\sum_{i=1}^{l} \sum_{j=1}^{q} \frac{c_{i j}}{\left(z-z_{i}\right)^{j}}+c_{0}
$$

with $l(\leq p)$ distinct poles of multiplicity $q$.

Each simply periodic solution is a rational function $R(\xi)$ of $\xi=e^{\alpha z}(\alpha \in \mathbf{C}) . R(\xi)$ has $l$ $(\leq p)$ distinct poles of multiplicity $q$, and it is of the form

$$
R(\xi)=\sum_{i=1}^{l} \sum_{j=1}^{q} \frac{c_{i j}}{\left(\xi-\xi_{i}\right)^{j}}+c_{0} .
$$

In order to give the representations of the elliptic solutions, we need some notation and results concerning the elliptic function [6].

Let $\omega_{1}, \omega_{2}$ be two given complex numbers such that $\operatorname{Im} \frac{\omega_{1}}{\omega_{2}}>0$, let $L=L\left[2 \omega_{1}, 2 \omega_{2}\right]$ be discrete subset $L\left[2 \omega_{1}, 2 \omega_{2}\right]=\left\{\omega \mid \omega=2 n \omega_{1}+2 m \omega_{2}, n, m \in \mathbf{Z}\right\}$, which is isomorphic to $\mathbf{Z} \times \mathbf{Z}$. The discriminant is $\Delta=\Delta\left(c_{1}, c_{2}\right):=c_{1}^{3}-27 c_{2}^{2}$ and

$$
s_{n}=s_{n}(L):=\sum_{n \geq 3, n \in N} \frac{1}{\omega^{n}} .
$$

The Weierstrass elliptic function $\wp(z):=\wp\left(z, g_{2}, g_{3}\right)$ is a meromorphic function with double periods $2 \omega_{1}, 2 \omega_{2}$, and satisfying the equation

$$
\left(\wp^{\prime}(z)\right)^{2}=4 \wp(z)^{3}-g_{2} \wp(z)-g_{3}
$$

where $g_{2}=60 s_{4}, g_{3}=140 s_{6}$ and $\Delta\left(g_{2}, g_{3}\right) \neq 0$.

On changing Eq. (11) to the form

$$
\left(\wp^{\prime}(z)\right)^{2}=4\left(\wp(z)-e_{1}\right)\left(\wp(z)-e_{2}\right)\left(\wp(z)-e_{3}\right),
$$

we have $e_{1}=\wp\left(\omega_{1}\right), e_{2}=\wp\left(\omega_{2}\right), e_{3}=\wp\left(\omega_{1}+\omega_{2}\right)$.

Inversely, given two complex numbers $g_{2}$ and $g_{3}$ such that $\Delta\left(g_{2}, g_{3}\right) \neq 0$, there exists a Weierstrass elliptic function $\wp(z)$ with double periods $2 \omega_{1}, 2 \omega_{2}$ such that the above holds.

Lemma $4[6,8]$ The Weierstrass elliptic functions $\wp(z):=\wp\left(z, g_{2}, g_{3}\right)$ have two successive degeneracies and in addition we have the following. 
(I) Degeneracy to simply periodic functions (i.e., rational functions of one exponential $\left.e^{k z}\right)$ occurs according to

$$
\wp\left(z, 3 d^{2},-d^{3}\right)=2 d-\frac{3 d}{2} \operatorname{coth}^{2} \sqrt{\frac{3 d}{2}} z
$$

if one root $e_{j}$ is double $\left(\Delta\left(g_{2}, g_{3}\right)=0\right)$.

(II) Degeneracy to rational functions of $z$ occurs according to

$$
\wp(z, 0,0)=\frac{1}{z^{2}}
$$

if one root $e_{j}$ is triple $\left(g_{2}=g_{3}=0\right)$.

(III) The addition formula holds according to

$$
\wp\left(z-z_{0}\right)=-\wp(z)-\wp\left(z_{0}\right)+\frac{1}{4}\left[\frac{\wp^{\prime}(z)+\wp^{\prime}\left(z_{0}\right)}{\wp(z)-\wp\left(z_{0}\right)}\right]^{2} .
$$

By the above lemmas, we can give a new method below, called, say, the complex method, to find exact solutions of some PDEs.

Step 1. Substituting the transform $T: u(x, y, t) \rightarrow w(z),(x, y, t) \rightarrow z$ into a given PDE gives a non-linear ordinary differential equation (5) or (7).

Step 2. Substitute Eq. (6) into Eq. (5) or (7) to determine whether the weak $\langle p, q\rangle$ condition holds.

Step 3. By the indeterminate relations (8)-(10) we find the elliptic, rational, and simply periodic solutions $w(z)$ of Eq. (5) or (7) with pole at $z=0$, respectively.

Step 4. By Lemmas 1 and 3 we obtain all meromorphic solutions $w\left(z-z_{0}\right)$.

Step 5. Substituting the inverse transform $T^{-1}$ into these meromorphic solutions $w\left(z-z_{0}\right)$, we get all exact solutions $u(x, t)$ of the originally given PDE.

\section{Proof of Theorem 1}

Substituting (6) into Eq. (1) we have $q=2, p=1, c_{-2}=\frac{12 \omega\left(k^{2}+l^{2}\right)}{k C_{R}}, c_{-1}=0, c_{0}=-1-\frac{\omega}{k C_{R}}$, $c_{1}=0, c_{2}=\frac{\left(\omega+k C_{R}\right)^{2}-2 d k C_{R}}{20 \omega\left(k^{2}+l^{2}\right) k C_{R}}, c_{3}=0, c_{4}$ is arbitrary.

Hence, Eq. (1) satisfies the weak $\langle 1,2\rangle$ condition and is a second-order Briot-Bouquet differential equation. Obviously, Eq. (1) satisfies the dominant condition. So, by Lemma 3, we know that all meromorphic solutions of Eq. (1) belong to $W$. Now we will give the forms of all meromorphic solutions of Eq. (1).

By Eq. (9), we infer that the indeterminate rational solutions of Eq. (1) with pole at $z=0$ have the form of

$$
R_{1}(z)=\frac{c_{2}}{z^{2}}+\frac{c_{1}}{z}+c_{10}
$$

Substituting $R_{1}(z)$ into Eq. (1), we get

$$
R_{1}(z)=\frac{12 \omega\left(k^{2}+l^{2}\right)}{k C_{R} z^{2}}-1-\frac{\omega}{k C_{R}} .
$$


Here $2 d k C_{R}=\left(\omega+k C_{R}\right)^{2}$. Thus for all rational solutions of Eq. (1)

$$
R(z)=\frac{12 \omega\left(k^{2}+l^{2}\right)}{k C_{R}\left(z-z_{0}\right)^{2}}-1-\frac{\omega}{k C_{R}}
$$

where $2 d k C_{R}=\left(\omega+k C_{R}\right)^{2}, z_{0} \in \mathbb{C}$.

In order to have simply periodic solutions, set $\xi=e^{\alpha z}$, put $w=R(\xi)$ into Eq. (1); then

$$
-\omega\left(k^{2}+l^{2}\right)\left[\xi R^{\prime}+\xi^{2} R^{\prime \prime}\right]+\left(w+k C_{R}\right) R+\frac{1}{2} k C_{R} R^{2}+d=0 .
$$

Substituting

$$
R_{2}(\xi)=\frac{c_{2}}{(\xi-1)^{2}}+\frac{c_{1}}{\xi-1}+c_{10}
$$

into Eq. (16), we obtain

$$
R_{2}(z)=\frac{12 \omega\left(k^{2}+l^{2}\right) \alpha^{2}}{k C_{R}(\xi-1)^{2}}+\frac{12 \omega\left(k^{2}+l^{2}\right) \alpha^{2}}{k C_{R}(\xi-1)}+\frac{\omega\left(k^{2}+l^{2}\right) \alpha^{2}}{k C_{R}}-1-\frac{\omega}{k C_{R}}
$$

Here $2 d k C_{R}=\left(\omega+k C_{R}\right)^{2}-\omega^{2}\left(k^{2}-l^{2}\right)^{2} \alpha^{2}$. Substituting $\xi=e^{\alpha z}$ into the above relation, and then we get simply periodic solutions of Eq. (1) with pole at $z=0$ :

$$
\begin{aligned}
w_{s 0}(z) & =\frac{12 \omega\left(k^{2}+l^{2}\right) \alpha^{2}}{k C_{R}\left(e^{\alpha z}-1\right)^{2}}+\frac{12 \omega\left(k^{2}+l^{2}\right) \alpha^{2}}{k C_{R}\left(e^{\alpha z}-1\right)}+\frac{\omega\left(k^{2}+l^{2}\right) \alpha^{2}}{k C_{R}}-1-\frac{\omega}{k C_{R}} \\
& =\frac{12 \omega\left(k^{2}+l^{2}\right) \alpha^{2} e^{\alpha z}}{k C_{R}\left(e^{\alpha z}-1\right)^{2}}+\frac{\omega\left(k^{2}+l^{2}\right) \alpha^{2}}{k C_{R}}-1-\frac{\omega}{k C_{R}} \\
& =w_{s}(z)=\frac{3 \omega\left(k^{2}+l^{2}\right)}{k C_{R}} \alpha \operatorname{coth}^{2} \frac{\alpha}{2}(z)-\frac{2 \omega\left(k^{2}+l^{2}\right)}{k C_{R}} \alpha^{2}-1-\frac{\omega}{k C_{R}} .
\end{aligned}
$$

So all simply periodic solutions of Eq. (1) are obtained by

$$
w_{s}(z)=\frac{3 \omega\left(k^{2}+l^{2}\right)}{k C_{R}} \alpha \operatorname{coth}^{2} \frac{\alpha}{2}\left(z-z_{0}\right)-\frac{2 \omega\left(k^{2}+l^{2}\right)}{k C_{R}} \alpha^{2}-1-\frac{\omega}{k C_{R}},
$$

where $2 d k C_{R}=-\omega^{2}\left(k^{2}+l^{2}\right)^{2} \alpha^{4}+\left(\omega+k C_{k}\right)^{2}, \alpha \neq 0, z_{0} \in \mathbb{C}$.

From Eq. (8) of Lemma 3, we have indeterminate relations of the elliptic solutions of Eq. (1) with pole at $z=0$,

$$
w_{d 0}(z)=c_{-2} \wp(z)+c_{0} .
$$

Putting $w_{d 0}(z)$ into Eq. (1), we obtain

$$
w_{d 0}(z)=\frac{12 \omega\left(k^{2}+l^{2}\right)}{k C_{R}} \wp(z)-1-\frac{\omega}{k C_{R}} .
$$

Here $42 d k C_{R}=-12 \omega^{2}\left(k^{2}+l^{2}\right)^{2} g_{2}+\left(\omega+k C_{k}\right)^{2}$. Therefore, for all elliptic solutions of Eq. (1)

$$
w_{d}(z)=\frac{12 \omega\left(k^{2}+l^{2}\right)}{k C_{R}} \wp\left(z-z_{0}\right)-1-\frac{\omega}{k C_{R}},
$$




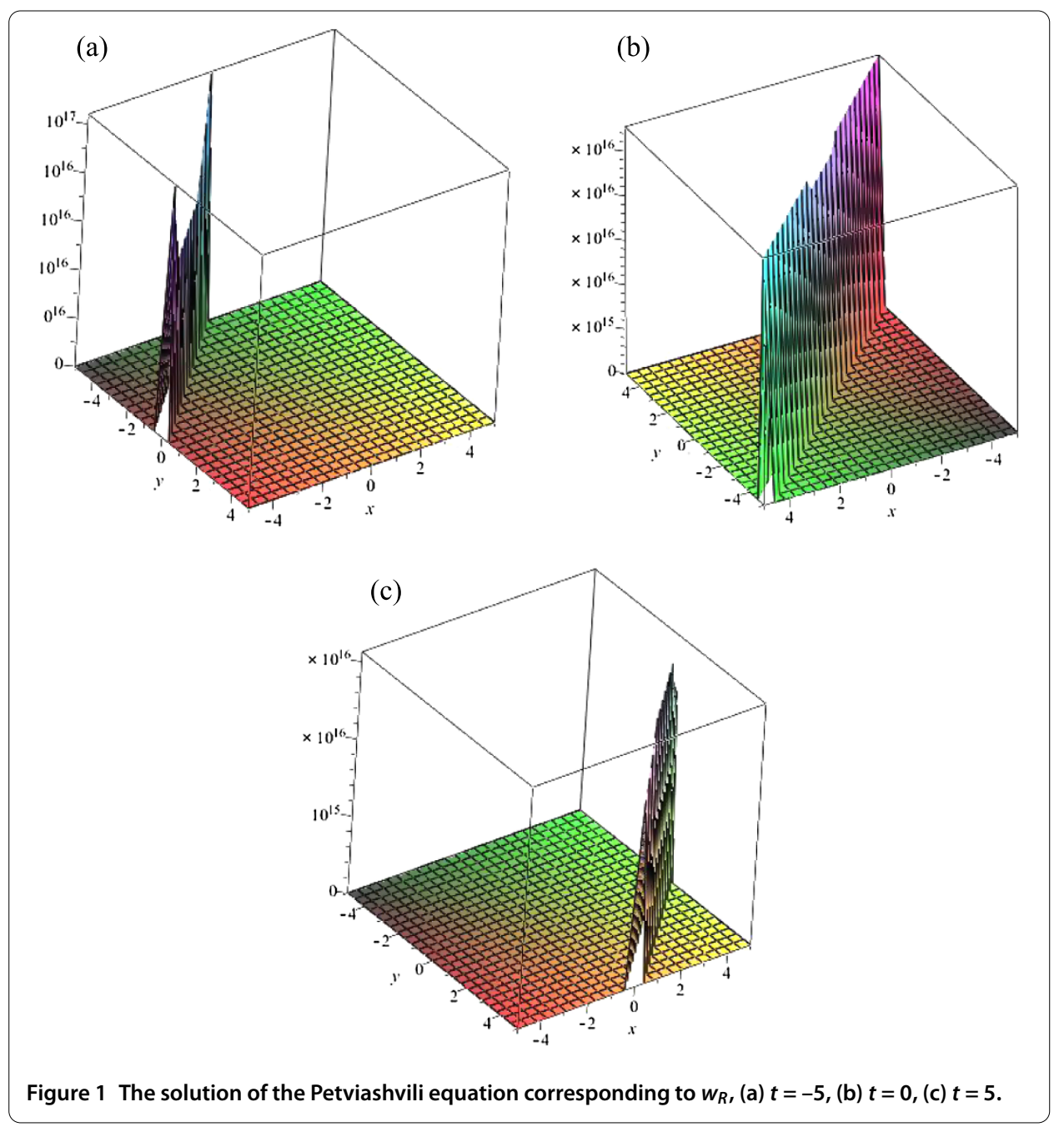

where $z_{0} \in \mathbb{C}$. Making use of the addition formula of Lemma 4, we rewrite it in the form

$$
\begin{aligned}
w_{d}(z)= & \frac{12 \omega\left(k^{2}+l^{2}\right)}{k C_{R}}\left\{-\wp(z)+\frac{1}{4}\left[\frac{\wp^{\prime}(z)+F}{\wp(z)-E}\right]^{2}\right\} \\
& -\frac{12 \omega\left(k^{2}+l^{2}\right) E}{k C_{R}}-1-\frac{\omega}{k C_{R}} .
\end{aligned}
$$

Here, $2 d k C_{R}=-12 \omega^{2}\left(k^{2}+l^{2}\right)^{2} g_{2}+\left(\omega+k C_{k}\right)^{2}, F^{2}=4 E^{3}-g_{2} E-g_{3}, g_{3}$, and $E$ are arbitrary.

\section{Computer simulations for new solutions}

In this section, we give some computer simulations to illustrate our main results. Here we take the new rational solutions $w_{R}(z)$ and simply periodic solutions $w_{s}(z)$ to further analyze their properties by Figures 1 and 2 .

(1) Take $k=l=1, \omega=C_{R}=-1, d=2, z_{0}=0$ in $w_{R}(z)$.

(2) Take $k=l=1, \omega=C_{R}=-1, d=2, z_{0}=0, \alpha=1$ in $w_{s}(z)$. 

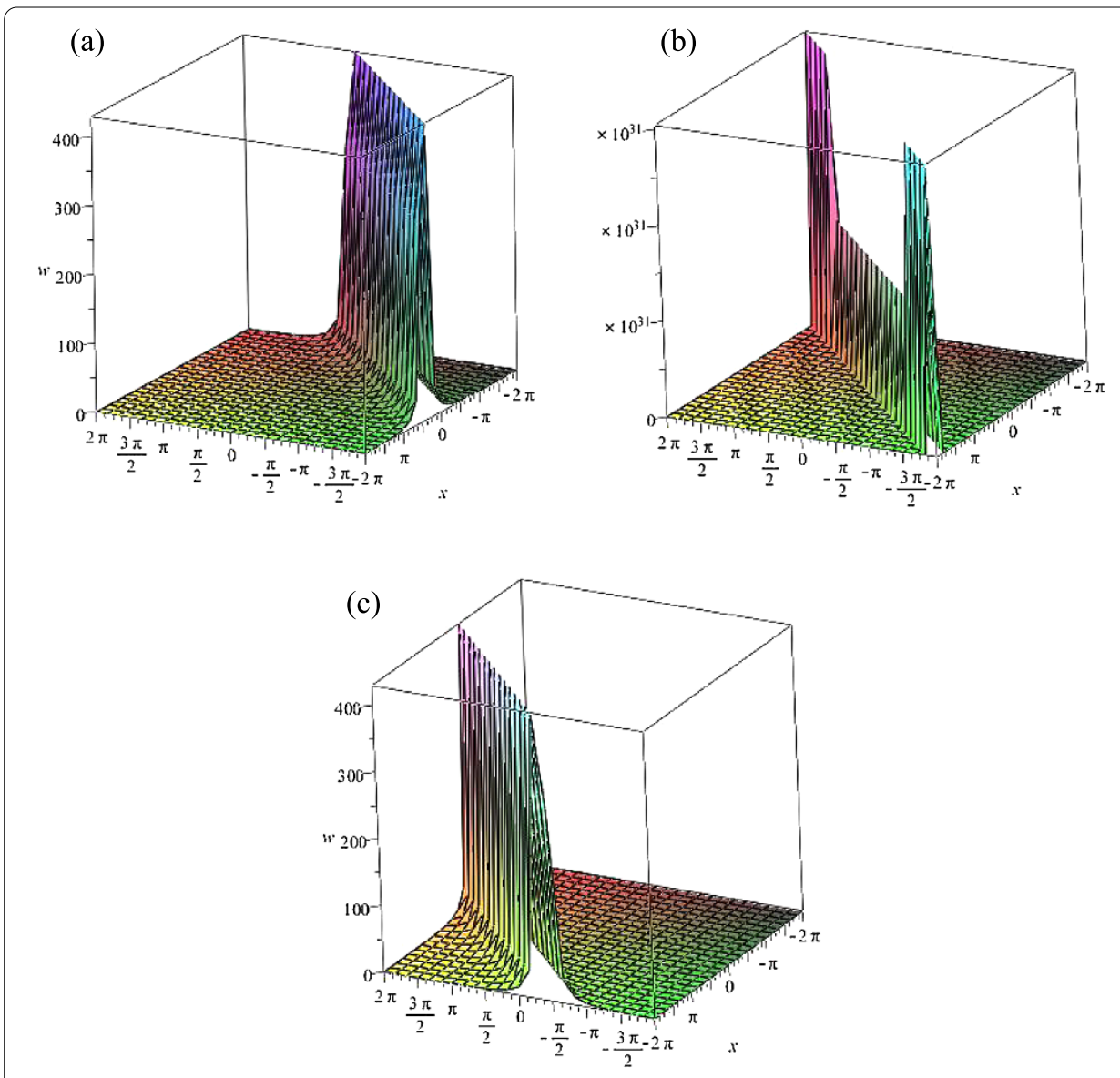

Figure 2 The solution of the Petviashvili equation corresponding to $w_{s}$, (a) $t=-5$, (b) $t=0$, (c) $t=5$.

\section{Conclusions}

The complex method is a very important tool in finding the traveling wave exact solutions of non-linear evolution equations such as the Petviashvili equation. In this paper, we employ the complex method to obtain all meromorphic exact solutions of the complex variant Eq. (1); then we find all traveling wave exact solutions of the Petviashvili equation. The idea introduced in this paper can be applied to other non-linear evolution equations. Our result shows that the complex method is simpler than other methods.

\section{Competing interests}

The authors declare that they have no competing interests.

Authors' contributions

ZH and WY carried out the design of the study and performed the analysis. JL participated in its design and coordination. All authors read and approved the final manuscript.

\section{Author details}

${ }^{1}$ School of Mathematics and Information Science, Guangzhou University, Guangzhou, 510006, China. ${ }^{2}$ Key Laboratory of Mathematics and Interdisciplinary Sciences of Guangdong Higher Education Institutes, Guangzhou University, Guangzhou, 510006, China.

\section{Acknowledgements}

This work is supported by the NSF of China (11271090) and NSF of Guangdong Province (S2012010010121). Also this work was supported by the Visiting Scholar Program of the Chern Institute of Mathematics at Nankai University where the authors worked as visiting scholars. The authors would like to express their hearty thanks to the Chern Institute of Mathematics providing very comfortable research environments to them. The authors finally wish to thank Professor 
Robert Conte for supplying his useful reprints and suggestions. The authors wish to thank the referees and editors for their very helpful comments and useful suggestions.

Received: 21 September 2013 Accepted: 19 December 2013 Published: 17 Jan 2014

\section{References}

1. Zhang, L, Zhang, Y, Liu, SK: Two-dimensional Rossby wave: exact solutions to Petviashvili equation. Commun. Theor. Phys. 45, 414-416 (2006)

2. Zhang, L, Zhang, LF, Li, CY, Wang, T, Tan, YK: Some new exact solutions of Jacobian elliptic function of Petviashvili equation. Commun. Theor. Phys. 49, 1557-1560 (2008)

3. Eremenko, A: Meromorphic solutions of equations of Briot-Bouquet type. Teor. Funktsii, Funk. Anal. i Prilozh. 38, 48-56 (1982). English translation: Transl. Am. Math. Soc. 133(2), 15-23 (1986)

4. Eremenko, A, Liao, LW, Ng, TW: Meromorphic solutions of higher order Briot-Bouquet differential equations. Math. Proc. Camb. Philos. Soc. 146(1), 197-206 (2009)

5. Kudryashov, NA: Meromorphic solutions of nonlinear ordinary differential equations. Commun. Nonlinear Sci. Numer. Simul. 15(10), 2778-2790 (2010)

6. Lang, S: Elliptic Functions, 2nd edn. Springer, New York (1987)

7. Yuan, WJ, Shang, YD, Huang, Y, Wang, H: The representation of meromorphic solutions of certain ordinary differential equations and its applications. Scientia Sinica Mathematica 43(6), 563-575 (2013). doi:10.1360/012012-159

8. Conte, R, Musette, M: Elliptic general analytic solutions. Stud. Appl. Math. 123(1), 63-81 (2009)

10.1186/1687-1847-2014-24

Cite this article as: Huang et al.: The general meromorphic solutions of the Petviashvili equation. Advances in Difference Equations 2014, 2014:24

\section{Submit your manuscript to a SpringerOpen ${ }^{\circ}$ journal and benefit from:}

- Convenient online submission

- Rigorous peer review

- Immediate publication on acceptance

- Open access: articles freely available online

- High visibility within the field

- Retaining the copyright to your article 